\title{
Sleep Quality for Patients Receiving Noninvasive Positive Pressure Ventilation and Nasal High-Flow Oxygen Therapy in an ICU: Two Case Studies
}

\author{
Hiroaki Murata1 ${ }^{*}$, Yoko Yamaguchi² $^{2}$ \\ ${ }^{1}$ School of Nursing at Narita, International University of Health and Welfare, Chiba, Japan \\ ${ }^{2}$ Department of Intensive Care Unit, Jikei University Hospital, Tokyo, Japan \\ Email: ^h.murata@iuhw.ac.jp
}

How to cite this paper: Murata, H. and Yamaguchi, Y. (2018) Sleep Quality for Patients Receiving Noninvasive Positive Pressure Ventilation and Nasal High-Flow Oxygen Therapy in an ICU: Two Case Studies. Open Journal of Nursing, 8, 605-615. https://doi.org/10.4236/ojn.2018.89045

Received: August 14, 2018

Accepted: September 8, 2018

Published: September 11, 2018

Copyright (c) 2018 by authors and Scientific Research Publishing Inc. This work is licensed under the Creative Commons Attribution International License (CC BY 4.0).

http://creativecommons.org/licenses/by/4.0/

\begin{abstract}
Aim: The purpose of this case study was to examine the sleep quality of patients receiving noninvasive positive pressure ventilation (NPPV) or nasal high-flow oxygen therapy (NHF) in an intensive care unit and to investigate what types of nursing support are offered to such patients. Methods: We examined one patient each for NPPV and NHF. Polysomnography (PSG), review of the patient charts, and semi-structured interviews were used to collect the data for analysis. Results: Patients treated with NPPV or NHF demonstrated a noticeable reduction in deep sleep, with most of their sleep being shallow. Their sleep patterns varied greatly from those of healthy individuals. These results suggest that, in addition to experiencing extremely fragmented sleep, sleep in these patients was more likely to be interrupted by nursing interventions, such as during auscultation of breath sounds. Furthermore, it was revealed that "anxiety or discomfort that accompanies the mask or air pressure" in patients treated with NPPV and "discomfort that accompanies the nasal cannula or NHF circuit" in patients treated with NHF may be primary causes of disrupted sleep. Our results suggest a need for nursing care aimed at improving sleep quality in patients treated with NPPV or NHF.
\end{abstract}

\section{Keywords}

Noninvasive Positive Pressure Ventilation (NPPV), Nasal High-Flow Oxygen Therapy (NHF), Sleep Deprivation, ICU, Post Intensive Care Syndrome (PICS) 


\section{Introduction}

The quality of sleep is impaired in critically ill patients [1] [2]. Sleep disorders are likely to be affected by endocrine function modulation [1], impaired immunity, cognitive function, pain [2], as well as discomfort [3] [4]. This is an issue that cannot be ignored by intensive care unit (ICU) medical teams providing support to critically ill patients.

It has been found that both environmental and physiological/psychological factors contribute to the development of sleep disorders in the ICU [5]. Consequently, in addition to creating a night-time environment inside the room that is dark and as quiet as possible, we are providing medical care that includes interventions focusing on relief of pain and anxiety, encouraging sleep with sleep-inducing drugs, and increasing the depth of sedation to a level higher than that used during the day, along with other nursing practices. However, it is true that numerous critically ill patients complain of lack of sleep.

Invasive respiratory therapy might cause sleep disturbance in the ICU. In contrast, noninvasive positive pressure ventilation (NPPV) and nasal high-flow oxygen therapy (NHF) are easier to introduce than intubation and are often less invasive and do not require analgesia and sedation. Therefore, patients treated with NPPV and NHF may be able to communicate verbally and sometimes eat and drink. Treatment with NPPV and NHF has been increasing in recent years as a form of respiratory management along with artificial-respiration management with conventional tracheal intubation in patients with acute respiratory failure. Conversely, critically ill patients who are treated with NPPV or NHF encounter several issues that may interfere with sleep, such as discomfort [6]. Some studies have suggested that insomnia during treatment with NPPV may lead to NPPV discontinuation [6]. Therefore, it is important to practice care for nurses who support the lifestyle and psychological wellbeing of critically ill patients.

There are several studies on the sleep conditions and sleep inhibitors in patients with tracheal intubation and oxygen mask/nasal cannula [7] [8] [9]. However, few studies have quantitatively and qualitatively examined the sleep conditions among patients treated with NPPV or NHF.

The purpose of this case study was to clarify the sleep conditions of patients receiving NPPV and NHF in the ICU and to examine the ideal manner of nursing support.

\section{Methods}

\subsection{Data Collection Period and Facilities}

This study was conducted between August 2014 and February 2015. The study took place in the ICU of a university hospital with 20 beds and a nurse to patient ratio of 1 to 2. 'A curtain or sliding door could be used to separate the patient's room from those of other patients or the ICU hallway. 


\subsection{Subjects}

1) Selection criteria

a) Adult patients $(-1 \leq$ RASS $\leq 1)$ who remained in the ICU for at least 1 night ( $9 \mathrm{pm}$ to $9 \mathrm{am}$ ) and were capable of communicating.

b) Patients who were treated with NPPV or NHF for the first time

2) Exclusion criteria

a) Patients after tracheal intubation in the ICU

b) The primary disease was cerebrovascular or neuromuscular

c) Patients who were assessed as having delirium based on the Confusion Assessment Method for the ICU [10] at the start of the study

d) Patients who had a psychiatric disorder and were receiving drug therapy for their condition

e) Patients with markedly poor circulatory dynamics and respiratory status

f) Patients continuously receiving analgesics, sedatives, and sleep introducers (drugs that may strongly affect the electroencephalogram (EEG))

\subsection{Data Collection}

1) Data collection with polysomnography (PSG)

The purpose of data collection with PSG was to determine the sleep stages and to accurately measure the presence and degree of arousal responses. Therefore, the three electrophysiological indicators essential for objective sleep measurements, EEG, electro-oculogram (EOG), and electromyogram (EMG), were measured. Generally, in PSG, information on oral/nasal respiration, respiratory movements of the chest and abdomen, percutaneous arterial blood oxygen saturation, electrocardiogram, tibialis anterior EMG, and position are simultaneously collected; however, taking into consideration the burden on the subjects, it was decided that these parameters would not be measured for the present study.

An Alice PDx (Philips Respironics, Murrysville, PA; medical equipment certification number: 22100BZX01027000), which is compact and can be used in the ICU, was used for measurements. Installation of the equipment was conducted by one project manager who had taken a lecture from a full-time laboratory technician from the Philips Respironics sleep center who was familiar with the standard method of the international EEG society (10 - 20 electrode system).

Subjects were at rest in a normal bed in the ICU. In the present study, "Lights out (in bed)" was defined as "lowering the lights in the ICU at approximately 9 pm" and "waking up" was defined as "when the patients naturally woke up in the morning" or "the beginning of medical care at approximately 7 am."

In accordance with the international 10 - 20 electrode system, the EEG installation sites for $\mathrm{A} 1$ and $\mathrm{A} 2$ were the earlobes (reference electrodes) attached to $\mathrm{C} 3$ and $\mathrm{O} 2$, and the body ground was placed on the forehead [11]. EOG electrodes were attached in two places: $1 \mathrm{~cm}$ below the lateral orbital rim and contralaterally $1 \mathrm{~cm}$ above the lateral orbital rim of the eye. EMG electrodes were attached at the left and right sides of the jaw [11]. 
2) Data collection by interview

A semi-structured interview was conducted by a researcher with a major theme such as "Sleep status during oxygen therapy (NPPV or NHF)" "How does oxygen therapy (NPPV or NHF) interfere with sleep?" and was able to add questions as appropriate for each patient. The interview took place in a quiet room where privacy could be assured and was recorded using a digital voice recorder with the permission of the subjects. The interview time was scheduled to be no more than 20 minutes per session, taking into account the burden on the subjects.

3) Collection of data from medical records

Following the completion of data collection with PSG, the following information was collected.

Age, the Acute Physiology and Chronic Health Evaluation II score [11], the medical history, the treatment details, the nursing care (gargling, suction, listening to breathing sounds, location confirmation of routes, and clothing changes), and the sleep environment.

\subsection{Data Analysis}

1) $P S G$

Data analysis was performed by a laboratory technician at the Philips Respironics Sleep Center. Without access to subject-identifying information, sleep stage analysis was conducted using the EEG, EOG, and EMG data. The sleep stage analysis was performed in accordance with the classifications proposed by the American Academy of Sleep Medicine in 2007 [12].

2) Semi-structured interview

The analysis was performed qualitatively with reference to the Modified Ground Theory Approach (M-GTA) [13]. M-GTA is a method of qualitative research that builds concepts based on data pertaining to phenomena. The analysis mainly comprises "constant comparative analysis," "theoretical sampling," and "theoretical saturation." We followed the concept of comparative analysis in limited data.

As a specific analysis procedure, the entire dataset was read and we focused on the sections related to the analysis theme: "Sleep status and sleep effects of patients receiving NPPV or NHF." "In the M-GTA, the minimum analytical unit is the concept. Each concept is created from several pieces of data known as variations. Variations are collected along the analysis theme" [14]. Moreover, we considered the interrelationships between the concepts and we created categories.

M-GTA usually collects and analyzes multiple cases. However, in this study, a subset of the M-GTA methods were utilized to more clearly present patient experiences.

\section{Ethical Considerations}

This study was conducted with approval from the ethics review committee of the 
research facility. The researchers explained the objectives of the study, freedom of withdrawal, and confidentiality of data content to the patients. It was also explained that the data collection would immediately stop if the patients became uncomfortable with the electrodes or if discomfort occurred during the data collection in the ICU. In addition, we took care to avoid burdening the patients physically and mentally during the interviews.

\section{Results}

\subsection{Summary of Subject Characteristics}

The study subjects were two male patients admitted to the ICU for medical treatment (Table 1). In addition, the agitation level was Richmond Agitation-Sedation Scale, 0 in both patients.

\subsection{Sleep Status Based on PSG}

The hypnogram (including awakening frequency/time) of the subjects and the time-dependent nursing care providedare illustrated in Figure 1 and Figure 2. The test data for night sleep PSG are shown in Table 2. The total sleep time of the subjects was 6 to 8 hours, and N1 was $28 \%$ to $34 \%$ and N2 was $57 \%$ to $62 \%$, whereas $\mathrm{N} 3$ ( $0 \%$ to $0.6 \%$ ) and REM (8\%) were almost absent.

Frequent awakening was observed up to $12 \mathrm{am}$ in the patient treated with NPPV (Subject A; Figure 1). Between 12 am and 6 am, fragmented sleep was observed at the timing of nursing intervention. In the patient treated with NHF (Subject B; Figure 2), relatively long fragmented sleep was observed between 11 $\mathrm{pm}$ and $4 \mathrm{am}$ at the timing of nursing intervention. In addition, it was found that the patient treated with NPPV had a higher frequency of awakening in 1 hour than the patient treated with NHF.

\subsection{Sleep Status as Assessed through the Interviews}

The results of the interviews with the two patients are presented in Table 3 and Table 4. Based on our analysis, in subject A (NPPV), three categories were created from eight concepts. In subject B (NHF), three categories were created from five concepts. Both patients treated with NPPV and NHF experienced discomfort associated with the equipment (e.g., the nasal cannula or the respiratory circuit) and with the air pressure, as well as insomnia due to the perceived discomfort.

\section{Discussion}

The study showed that the sleep conditions in the examined patients treated with NPPV or NHF were markedly impaired, with a significant decrease in deep sleep (N3), and the presence of shallow sleep (N1 + N2) accounted for approximately $91 \%$ of sleep structure. The patients' sleep patterns varied greatly from those of healthy individuals. Moreover, these results suggest that, in addition to experiencing extremely fragmented sleep, sleep in these patients was more likely 
to be interrupted by nursing interventions, such as during auscultation of breath sounds. Furthermore, it was revealed that "anxiety or discomfort associated with the mask or air pressure" in the patient treated with NPPV and "discomfort associated with the nasal cannula or NHF circuit" in the patient treated with NHF could have been the primary causes of disrupted sleep. Thus, in this study, we were able to provide information regarding the sleep disorders and their probable causes in patients treated with NPPV or NHF.

The sleep structure of the patients treated with NPPV or NHF presented in this study was similar to that of patients treated with invasive ventilation using sedatives and in oxygen mask/nasal cannula [8] [9]. However, it was found that the frequency of awakening in an hour in the patient treated with NPPV, at 34.5 times, was higher than that reported by previous studies (frequency of awakening in 1 hour 27 times on average) [8] including patients treated with invasive ventilation and oxygen mask/nasal cannula. In contrast, the frequency of awakening
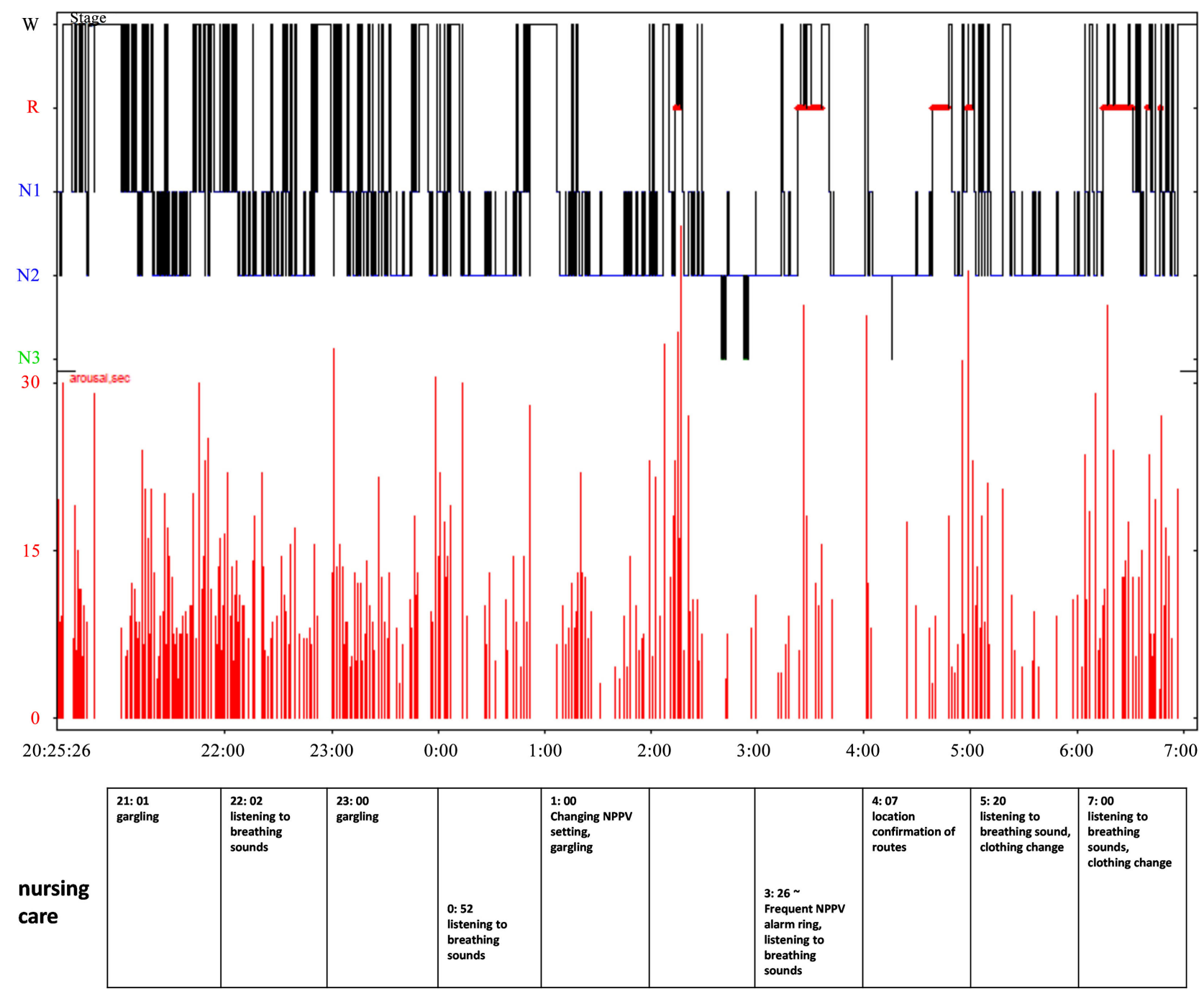

Figure 1. Hypnogram of the patient treated with NPPV (Subject A) and nursing care. W: wake, R: REM sleep, N1: nonREM sleep stage 1, N2: nonREM sleep stage 2, N3: nonREM sleep stage 3. 


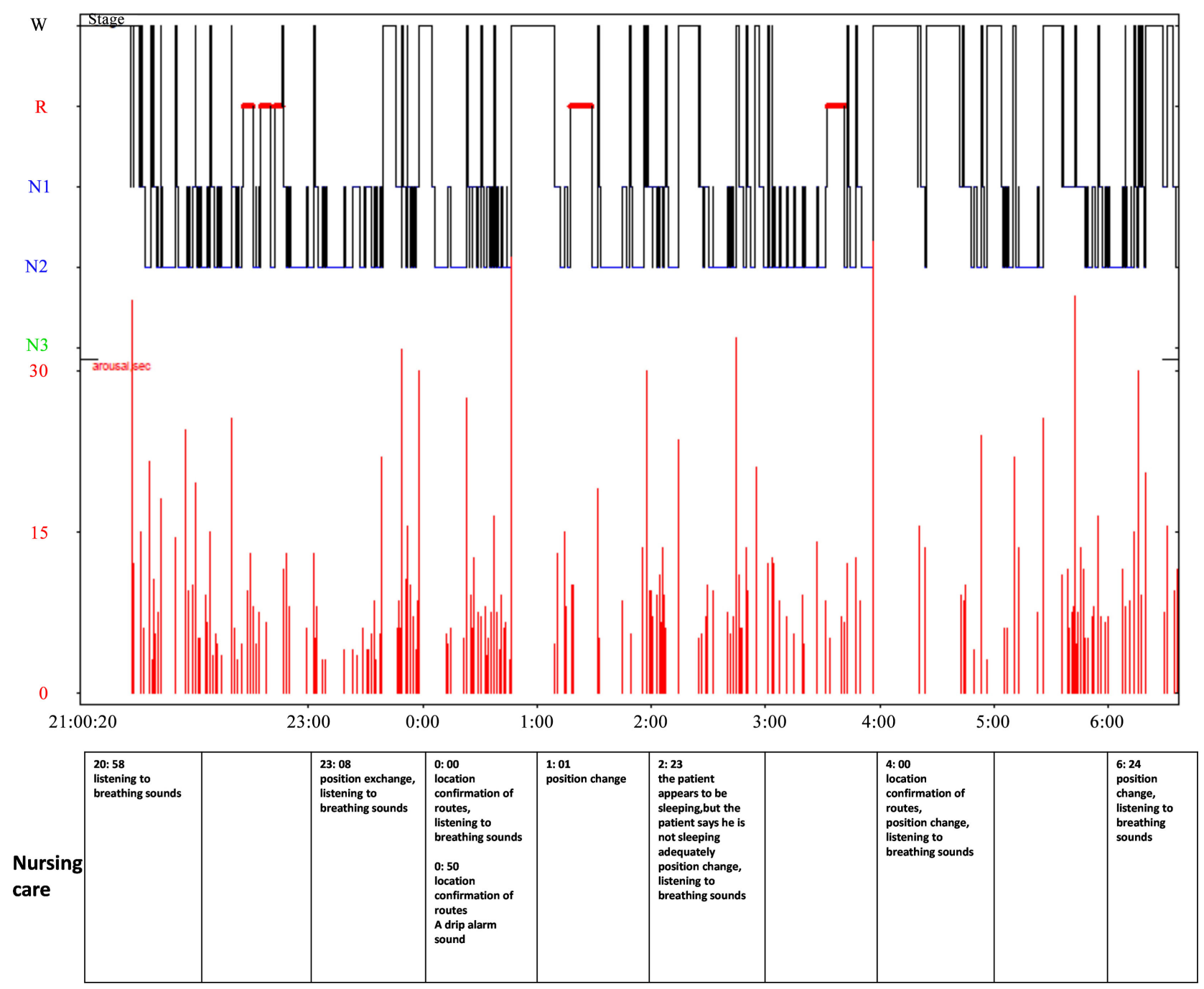

Figure 2. Hypnogram of the patient treated with NHF (Subject B) and nursing care. W: wake, R: REM sleep, N1: nonREM sleep stage 1, N2: nonREM sleep stage 2, N3: nonREM sleep stage 3.

Table 1. Participant characteristics.

\begin{tabular}{|c|c|c|c|c|c|c|c|c|c|}
\hline Participant & sex & age & $\begin{array}{l}\text { APACHE } \\
\text { II score }\end{array}$ & $\begin{array}{c}\text { respiratory } \\
\text { management }\end{array}$ & $\begin{array}{c}\text { Reason for starting } \\
\text { NPPV/NHF }\end{array}$ & Treatment & $\begin{array}{l}\text { Data Collection } \\
\text { with PSG }\end{array}$ & $\begin{array}{l}\text { Semi-structured } \\
\text { interview }\end{array}$ & $\begin{array}{l}\text { Interview } \\
\text { time }\end{array}$ \\
\hline A & $\operatorname{man}$ & $50 \mathrm{~s}$ & 24 & $\begin{array}{c}\text { NPPV } \\
\text { (mouth mask) }\end{array}$ & pulmonary edema & medical & NPPV and NHF & $\begin{array}{c}9 \text { days after NPPV } \\
\text { withdrawal }\end{array}$ & 28 minutes \\
\hline B & $\operatorname{man}$ & $70 \mathrm{~s}$ & 24 & NHF & acute pneumonia & medical & first day night & $\begin{array}{c}8 \text { days after NHV } \\
\text { withdrawal }\end{array}$ & 26 minutes \\
\hline
\end{tabular}

APACHE: Acute Physiologic Assessment and Chronic Health Evaluation [11], NPPV: noninvasive positive pressure ventilation, NHF: nasal high-flow oxygen therapy.

Table 2. Results of polysomnography.

\begin{tabular}{ccc}
\hline Test items of PSG & NPPV (Subject A) & NHF (Subject B) \\
\hline Total sleep time (TST) & 8.2 hours & 6.7 hours \\
\% Stage REM (\% TST) & $8.4 \%$ & $8.8 \%$ \\
\hline
\end{tabular}




\section{Continued}

\begin{tabular}{ccc} 
\% Stage N1 (\% TST) & $28.4 \%$ & $34.0 \%$ \\
\% Stage N2 (\% TST) & $62.6 \%$ & $57.2 \%$ \\
\% Stage N3 (\% TST) & $0.6 \%$ & $0.0 \%$ \\
Arousal index (times/hour) & 34.5 & 28.7 \\
\hline
\end{tabular}

N1: non-REM sleep stage 1, N2: nonREM sleep stage 2, N3: NonREM sleep stage 3, NPPV: noninvasive positive pressure ventilation, NHF: nasal high-flow oxygen therapy, Arousal index was defined as the number of arousals per hour during the Total sleep time (TST).

Table 3. Sleep status and sleep inhibitory factors of the patient treated with noninvasive positive pressure ventilation (NPPV).

\begin{tabular}{cl}
\hline Category & Concept \\
\hline & Feeling blockage and pressure by the mask was uncomfortable. \\
Thxiety and discomfort & The contact with the mask was painful. \\
associated with masks & I avoided moving because I was worried that the mask would be displaced. \\
& I wondered if air was leaking. \\
Discomfort associated & Feeling my mouth dry due to the strong wind pressure was uncomfortable. \\
with air pressure & The NPPV exerts strong wind pressure and made me feel uncomfortable. \\
Insomnia with anxiety or & I could not sleep because of anxiety or discomfort caused by the mask and wind pressure. \\
discomfort & Because the mask worried me and I had difficulty moving, my sleep was fragmented.
\end{tabular}

Table 4. Sleep status and sleep inhibitory factors of the patient treated with nasal high-flow oxygen therapy (NHF).

\begin{tabular}{|c|c|}
\hline Category & Concept \\
\hline & The contact with the cannula (nose and ear) was painful. \\
\hline $\begin{array}{l}\text { Discomfort associated } \\
\text { with the nasal cannula or } \\
\text { respiratory circuit }\end{array}$ & $\begin{array}{l}\text { Because the respiratory circuit was inflexible and could be easily entangled, I felt } \\
\text { uncomfortable moving. }\end{array}$ \\
\hline & I worried about the noise and vibration when water accumulated in the respiratory circuit. \\
\hline $\begin{array}{l}\text { Discomfort associated } \\
\text { with air pressure }\end{array}$ & $\begin{array}{l}\text { Feeling dryness in the nasal cavity and throat due to the strong wind pressure was } \\
\text { uncomfortable. }\end{array}$ \\
\hline Insomnia with discomfort & $\begin{array}{l}\text { Because the respiratory circuit worried me and I had difficulty moving, my sleep was } \\
\text { fragmented. }\end{array}$ \\
\hline
\end{tabular}

in an hour in the patient treated with NHF, at 28.7 times, was found to be similar to that previously reported. As a reason for this, a qualitative study showed that patients treated with NPPV were more uncomfortable and anxious than patients treated with NHF. This frequent arousal may lead to insomnia.

Based on the above, we consider nursing care for promoting sleep in patients treated with NPPV or NHF pertinent to the following two dimensions.

1) Nursing support for insomnia according to discomfort.

Both patients treated with NPPV and NHF may experience "discomfort due to difficulty in mobility" as indicated by the qualitative results. Medical teams including nurses need to be aware that patients treated with NPPV or NHF have difficulty moving and to properly explain to patients the extent of possible movement. This can help relieve patient discomfort and provide nursing support 
for sleep promotion.

In addition, in patients treated with NPPV, the respiratory alarm sounds when the mask or the respiratory circuit comes off, so we can respond quickly; for instance, to reposition them. In contrast, because the NHF does not have an alarm function, and therefore the medical personnel cannot be immediately alerted of abnormal respiratory events. Therefore, we need to convey the importance and necessity of the oxygen therapy to the patient. By doing this, we may be able to support the patient's willingness to retain the equipment in place, even though NPPV and NHF are unpleasant [6] [15]. This can promote safety and alleviate anxiety and thus promote sleep.

2) Nursing support for fragmented sleep

In this study, as in other studies, it was suggested that nursing interventions and the ICU environment may result in fragmented sleep [7] [16]. Therefore, nursing care should be carried out for patients treated with NPPV and NHF, as well as in the Pain, Agitation and Delirium guidelines [17], with the aim of minimizing disturbance during the night to maintain the patient's sleep cycle to the greatest possible extent.

Finally, sleep disturbances, including fragmented sleep, may be a risk factor for delirium [18]. Furthermore, delirium is a risk factor for post intensive care syndrome [19]. Therefore, improving sleep disorders, including fragmented sleep, is an important nursing-support consideration that can contribute to the long-term prognosis and improvement of health-related quality of life starting at the ICU, and it is necessary to continue research in patients treated with NPPV or NHF.

\section{Limitations}

There are several limitations in this study. First, we only studied one patient treated with NPPV and one with NHF. Which may have resulted in selection bias. In addition, from the medical records, we collected data, such as nursing care and alarm sounds, that were directly concerning these patients. However, there is a possibility that the records were not sufficiently documented and there may be information bias. In the future, it will be necessary to conduct prospective data collection and to increase the number of subjects in order to advance nursing care related to sleep deprivation and sleep promotion. However, we believe that this case study provided important information regarding the sleeping conditions of patients treated with NPPV or NHF, as we used PSG, which is the gold standard for sleep examination.

\section{Conclusions}

Patients treated with NPPV or NHF demonstrated a noticeable reduction in deep sleep, as most of their sleep was shallow. Their sleep patterns varied greatly from those of healthy individuals. These results suggest that, in addition to experiencing extremely fragmented sleep, sleep in these patients was more likely to 
be interrupted by nursing interventions, such as during auscultation of breath sounds.

Furthermore, it was revealed that "anxiety or discomfort that accompanies the mask or air pressure" in patients treated with NPPV and "discomfort that accompanies the nasal cannula or NHF circuit" in patients treated with NHF may be primary causes of disrupted sleep. This suggests a need for nursing care aimed at improving sleep quality in patients treated with NPPV or NHF.

\section{Acknowledgements}

We would like to express our deepest appreciation to the patients and hospital officials who cooperated in this research.

\section{Conflicts of Interest}

The authors declare no conflicts of interest regarding the publication of this paper.

\section{References}

[1] Matthews, E.E. (2011) Sleep Disturbances and Fatigue in Critically Ill Patients. AACN Advanced Critical Care, 22, 204-224. https://doi.org/10.1097/NCI.0b013e31822052cb

[2] Friese, R.S. (2008) Sleep and Recovery from Critical Illness and Injury: A Review of Theory, Current Practice, and Future Directions. Critical Care Medicine, 36, 697-705. https://doi.org/10.1097/CCM.0B013E3181643F29

[3] Nelson, J.E., Meier, D.E., Oei, E.J., Nierman, D.M., Senzel, R.S., Manfredi, P.L., Davis, S.M. and Morrison, R.S. (2001) Self-Reported Symptom Experience of Critically Ill Cancer Patients Receiving Intensive Care. Critical Care Medicine, 29, 277-282. https://journals.lww.com/ccmjournal/Fulltext/2001/02000/Self_reported_symptom_ experience_of_critically_ill.10.aspx https://doi.org/10.1097/00003246-200102000-00010

[4] Takashima, N., Murata, H., Nishikaichi, Y., Yamaguchi, Y., Sakaki, K. and Takinami, M. (2017) Stressful Experiences in the ICU of Patients Put on Mechanical Ventilators for 12 Hours or More. Journal of the Japanese Society of Intensive Care Medicine, 24, 399-405. (in Japanese) https://doi.org/10.3918/jsicm.24_399

[5] Pisani, M.A., Friese, R.S., Gehlbach, B.K., Schwab, R.J., Weinhouse, G.L. and Jones, S.F. (2015) Sleep in the Intensive Care Unit. American Journal of Respiratory and Critical Care Medicine, 191, 731-738. https://doi.org/10.1164/rccm.201411-2099CI

[6] Murata, H., Inoue, T. and Takahashi, O. (2017 What Prevents Critically Ill Patients with Respiratory Failure from Using Non-Invasive Positive Pressure Ventilation: A Mixed-Methods Study. Japan Journal of Nursing Science, 14, 297-310. https://doi.org/10.1111/jjns.12159

[7] Yamaguchi, A., Sugie, E., Hirao, A. and Egawa, K. (2017) Relationships between Nursing Interventions and Fragmentation of Sleep in Mechanically Ventilated ICU Patients. Journal of Japan Academy of Critical Care Nursing, 13, 65-70. (In Japanese). https://doi.org/10.11153/jaccn.13.1_65

[8] Elliott, R., McKinley, S., Cistulli, P. and Fien, M. (2013) Characterisation of Sleep in Intensive Care Using 24 Hour Polysomnography: An Observational Study. Critical 
Care, 17, R46. https://doi.org/10.1186/cc12565

[9] Freedman, N.S., Gazendam, J., Levan, L., Pack, A.I. and Schwab, R.J. (2001) Abnormal Sleep/Wake Cycles and the Effect of Environmental Noise on Sleep Disruption in the Intensive Care Unit. American Journal of Respiratory and Critical Care Medicine, 163, 451-457. https://doi.org/10.1164/ajrccm.163.2.9912128

[10] Koga, Y., Tsuruta, R., Murata, H., Matsuo, K., Ito, T., Ely, E.W., Shintani, A., Wakamatsu, H., Sanui, M. and Yamase, H. (2015) Reliability and Validity Assessment of the Japanese Version of the Confusion Assessment Method for the Intensive Care Unit (CAM-ICU). Intensive \& Critical Care Nursing. The Official Journal of the British Association of Critical Care Nurses, 31, 165-170. https://doi.org/10.1016/j.iccn.2014.10.002

[11] Knaus, W.A., Draper, E.A., Wagner, D.P. and Zimmerman, J.E. (1985) APACHE II: A Severity of Disease Classification System. Critical Care Medicine, 13, 818-829. https://doi.org/10.1097/00003246-198510000-00009

[12] Berry, R.B., Brooks, R., Gamaldo, C.E., Harding, S.M., Lloyd, R.M., Marcus, C.L. and Vaugh, B.V. (2015) The AASM Manual for the Scoring of Sleep and Associated Events: American Academy of Sleep Medicine. https://aasm.org/resources/pdf/scoring-manual-preface.pdf

[13] Kinoshita, Y. (2007) Modified Grounded Theory Approach. Kobundo, Tokyo, 15-303. (In Japanese).

[14] Odachi, R., Tamaki, T., Ito, M., Okita, T., Kitamura, Y. and Sobue, T. (2017) Nurses' Experiences of End-of-Life Care in Long-Term Care Hospitals in Japan: Balancing Improving the Quality of Life and Sustaining the Lives of Patients Dying at Hospitals. Asian Nursing Research, 11, 207-215. https://doi.org/10.1016/j.anr.2017.08.004

[15] Murata, H. and Inoue, T. (2011) Clinical Judgment of Nurses to Continue Noninvasive Positive-Pressure Ventilation (NPPV) in Patients with Acute Respiratory Failure. Journal of Japan Academy of Critical Care Nursing, 7, 36-44. (In Japanese). https://doi.org/10.11153/jaccn.7.1_36

[16] Bihari, S., Doug McEvoy, R., Matheson, E., Kim, S., Woodman, R.J. and Bersten, A.D. (2012) Factors Affecting Sleep Quality of Patients in Intensive Care Unit. Journal of Clinical Sleep Medicine, 8, 301-307.

[17] Barr, J., Fraser, G.L., Puntillo, K., Ely, E.W., Gelinas, C., Dasta, J.F., Davidson, J.E., Devlin, J.W., Kress, J.P., Joffe, A.M., Coursin, D.B., Herr, D.L., Tung, A, Robinson, B.R., Fontaine, D.K., Ramsay, M.A., Riker, R.R., Sessler, C.N., Pun, B., Skrobik, Y. and Jaeschke, R. (2013) Clinical Practice Guidelines for the Management of Pain, Agitation, and Delirium in Adult Patients in the Intensive Care Unit. Critical Care Medicine, 41, 263-306. https://doi.org/10.1097/CCM.0b013e3182783b72

[18] Weinhouse, G.L., Schwab, R.J., Watson, P.L., Patil, N., Vaccaro, B., Pandharipande, P. and Ely, E.W. (2009) Bench-to-Bedside Review: Delirium in ICU Patients-Importance of Sleep Deprivation. Critical Care, 13, 234. https://doi.org/10.1186/cc8131

[19] Needham, D.M., Davidson, J., Cohen, H., Hopkins, R.O., Weinert, C., Wunsch, H., et al. (2012) Improving Long-Term Outcomes after Discharge from Intensive Care Unit: Report from a Stakeholders' Conference. Critical Care Medicine, 40, 502-509. https://doi.org/10.1097/CCM.0b013e318232da75 